\title{
A Solution for the Treatment of a Neglected Subset of Rare Diseases
}

\author{
Grant E Fisher \\ University of North Carolina at Greensboro, USA
}

*Corresponding author: Grant E Fisher, University of North Carolina at Greensboro, North Carolina, USA

\begin{abstract}
A subset of rare disease defined hereunto is presented along with a solution to the neglect of development of therapies for this subset.
\end{abstract}

\section{Keywords}

Rare disease, Neglected rare disease, Commercialization

\section{Main Text}

"How many thousands of people suffered needlessly during the forty-six years between Gerhardt's discovery and Bayer's distribution of aspirin?" Eli Lilly opines in Thea Cooper's book Breakthrough [1]. It is no secret pharmaceutical companies take time to commercialize new therapeutics, sometimes for good reason and sometimes for no reason. There is a current subset of disease that has gone wholly ignored by the pharmaceutical industry. This is due to uncertainty about government regulation, uncertainty about insurance coverage, concerns over ethics, but namely a concern about a financial return.

Hyper-rare diseases (HRD) are a subset of rare diseases with a prevalence of less than 1 in 1,000,000. HRDs represent a broad variety of diseases, encompassing $84.5 \%$ of all rare diseases [2]. Pharmaceutical companies neglect hyper-rare disease, as such little-tono treatments have been commercially developed for HRDs.

HRDs are neglected by pharmaceutical companies due to assumptions about the commercial aspects of these diseases. But pharmaceutical companies are also neglecting the solution that will allow commercialization of therapies for HRDs: Charging prices high enough to recoup $R \& D$ expenses and profit.

Individual HRDs are made up of small patient populations, thus the high individual prices will amount to a relatively small total cost to insurers and the healthcare system overall. Treatments for HRDs will, in almost all cases, represent the first and only available treatments. As the collective price will not be problematic and with treatments being the first available, insurers will have an incentive to cover the prices necessary to develop and commercialize these therapies.

If the public is to accept this, the only proposed solution, they must realize HRDs are different from other rare diseases, and if they are to be treated, typical methods of evaluation such as cost-effectiveness should not be considered.

Pharmaceutical companies neglect the development of therapies, as countless examples prove, with, many times, little reason besides oft misplaced financial concerns. Pharmaceutical companies' timeliness has left many vulnerable populations wholly neglected and without any treatment options. The absence of and neglect of development of therapies for HRD's presents a clear solution.

\section{Competing Interests}

The authors declare that they have no competing interests.

\section{Funding}

No funding was received.

\section{Acknowledgements}

Not Applicable. 


\section{References}

1. Cooper T, Ainsberg A (2010) Breakthrough: Elizabeth Hughes, the discovery of insulin, and the making of a medical miracle. St Martin's Press, New York, 123.
2. Wakap SG, Lambert DM, Olry A, Rodwell C, Gueydan C, et al. (2020) Estimating cumulative point prevalence of rare diseases: Analysis of Orphanet database. Eur J Hum Genet 28: 165-173. 